\title{
О ВОЗМОЖНОСТИ ЭЛЕКТРОН-ФОНОННОГО МЕЖЗОННОГО МЕХАНИЗМА ВЫСОКОТЕМПЕРАТУРНОИ СВЕРХПРОВОДИМОСТИ
}

\author{
(Представил В. Хижняков)
}

Предложен возможный механизм высокотемпературной сверхпроводимости, основанный на электрон-фононном взанмодействии перекрывающихся дырочных зон проводимости.

Пионерской относительно получения высокотемпературной сверхпроводимости была работа К. А. Мюллера и Я. Г. Беднорца $\left[{ }^{1}\right]$ на системах типа (Ba, $\mathrm{La})_{2} \mathrm{CuO}_{4}$. Дальнейшее повышение температуры сверхпроводящего перехода $\left(T_{c}\right)$ в район $100 \mathrm{~K}$ достигнуто в классе систем $\mathrm{Y}_{1} \mathrm{Ba}_{2} \mathrm{Cu}_{3} \mathrm{O}_{7-\delta} \cdot\left[{ }^{2,3}\right]$. Несмотря на дальнейший и, видимо, значительный прогресс в получении все более высокотемпературной сверхпроводимости, в данном сообщении мы будем ориентироваться лишь на два упомянутых класса соединений. Әто связано с основной целью нашей работы - предложить один из возможных механизмов высокотемпературной сверхпроводимости, основываясь на особенностях электронного спектра данных систем. Расчеты одноэлектронного энергетического спектра для $\mathrm{La}_{2} \mathrm{CuO}_{4}$ выполнены в $\left[{ }^{4-7}\right]$, а для $\mathrm{Y}_{1} \mathrm{Ba}_{2} \mathrm{Cu}_{3} \mathrm{O}_{7}$ в $\left[{ }^{8-10}\right]$.

Выяснение механизма высокотемпературной сверхпроводимости задача очевидных интереса и значимости, и ей посвящено уже большое число разнообразных по физическому содержанию и различных по глубине работ. Возможности традиционного механизма БҚШ были, повидимому, наиболее объективно оценены в этом аспекте в [ $\left.{ }^{11}\right]$, не позволяя выйти в область с $T_{c}>40 \mathrm{~K}$. Поэтому большинство исследователей склонны к рассмотрению принципиально новых механизмов с различными электронными (нефононными) возбуждениями в качестве посредников сверхпроводящего спаривания, или вносящих тем или иным приемом шкалу электронных энергий как затравочную при определении $T_{c}$. В качестве иллюстративной (но достаточно случайной) выборки можно указать на работы $\left[{ }^{12-23}\right]$. Несмотря на обилие оптимистических оценок относительно величины $T_{c}$, вопрос о природе высокотемпературной сверхпроводимости к моменту написания настоящей заметки полностью не решен.

Нам представляется, что способность к высокотемпературной сверхпроводимости должна быть связана с некими характерными свойствами электронного энергетического спектра соответствующих материалов. При этом в первую очередь внимание следует обратить на валентные зоны (дырочные зоны проводимости), так как экспериментально установлено, что сверхпроводимость в обсуждаемых материалах обязана носителям с положительным знаком заряда $[24,25]$. В этом контексте сразу выясняется, что незаполненная часть «валентной» зоны (со спектром двухмерного типа генеалогически определяемым

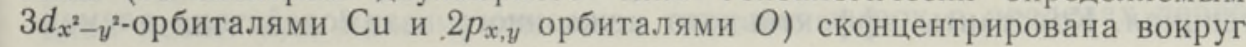


$X$-точки зоны Бриллюэна в $\mathrm{La}_{2} \mathrm{CuO}_{4}$ и $S$-точки в $\mathrm{Y}_{1} \mathrm{Ba}_{2} \mathrm{Cu}_{3} \mathrm{O}_{7}$. При этом имеет место перекрывание нескольких зон. Грубо говоря, максимум более низкой зоны лежит под максимумом верхней, а поверхность Ферми проходит, практически, по потолку нижней зоны в «нелегированном» соединении $\left(\mathrm{La}_{2} \mathrm{CuO}_{4}\right.$ без примеси $\mathrm{Ba}$ или $\mathrm{Y}_{1} \mathrm{Ba}_{2} \mathrm{Cu}_{3} \mathrm{O}_{7-\delta}$ с $\delta=0)$, которое еще не показывает свойства сверхпроводника. В этой связи встает вопрос о возможной роли межзонного электрон-фононного взаимодействия как причины сверхпроводящего спаривания, Это, конечно, означает отход от традиционной схемы БКШ, оперирующей с эффективным электронным притяжением в пределах одной зоны. В схеме БКШ соответственно появляется фононный масштаб энергий для обеспечения нужного знака взаимодействия. В случае же межзонного электрон-фононного взаимодействия снимается жесткое требование относительно знака взаимодействия и соответственно могут «рабо-

тать» значительные участки $\vec{k}$-пространства, т. е. появляется электронный масштаб энергий. О возможной роли нескольких зон говорит также то, что в ряде случаев в эксперименте наблюдаются две сверхпроводящие щели $\left[{ }^{26}\right]$.

Кроме того, о возможной роли межзонного электрон-фононного взаимодействия можно вспомнить и в связи с вибронной теорией сегнетоэлектричества [ $\left.{ }^{27,28}\right]$. Высокотемпературные сверхпроводники в известной мере родственны соединениям $\mathrm{SrTiO}_{3}$ и ( $\left.\mathrm{Ba}, \mathrm{Bi}\right) \mathrm{PbO}_{3}\left[{ }^{29}\right]$, являющимися одновременно сегнетоэлектриками и родоначальниками ряда веществ, исследование которых привело к резко возрастающей зависимости обнаруженных $T_{c}$ от времени. В то время как внутризонные электрон-фононные взаимодействия в системах низкой размерности могут привести к пайерлсовскому переходу, межзонное электронфононное взаимодействие может привести к структурным фазовым переходам типа сегнетоэлектрических. «Сегнетоэлектрическая тенденция» проявляется и у высокотемпературных сверхпроводников, в частности, y $\mathrm{La}_{2} \mathrm{CuO}_{4}$ имеется структурный переход из тетрагональной (несверхпроводящей) фазы в орторомбическую, причем наблюдаема и соответствующая мягкая мода $\left[{ }^{30}\right]$.

Отметим, что электрон-фононный механизм сверхпроводимости в связи с участием несколькнх зон уже затрагивался в литературе: [ $\left.{ }^{31}\right]$ (несколько $30 н) ;\left[{ }^{32}\right]$ (несколько долин); $\left[{ }^{33}\right]$ (зона и локальные состояния).

Начнем с того, что выпишем гамильтониан, получающийся путем обычной фрелиховской процедуры введения эффективного, обусловленного электрон-фононной связью взаимодействия носителей многозонной модели. В целях простоты опускаем вклады, обусловленные внутризонным взаимодействием и частью межзонного взаимодействия, формирующего пары на разных зонах. В итоге сохраняется межзонное взаимодействие с «однозонными» парами и гамильтониан имеет вид

$$
\begin{aligned}
& H=\sum_{\sigma, \vec{k}, s}\left[\varepsilon_{\sigma}(\vec{k})-\mu\right] \underset{\sigma \overrightarrow{k s}}{a_{\sigma k s}^{+}} a_{\sigma \vec{k}}+ \\
& +2 \sum_{\sigma, \sigma^{\prime}} \sum_{\vec{k}, \vec{k}^{\prime}} W_{\sigma \sigma^{\prime}}\left(\overrightarrow{k,}, \overrightarrow{k^{\prime}}\right) a_{\sigma \vec{k} \uparrow}^{+} a_{\sigma-\vec{k} \downarrow}^{+} a_{\sigma^{\prime}-\vec{k}^{\prime} \downarrow} a_{\sigma^{\prime} \overrightarrow{k^{\prime} \uparrow}}
\end{aligned}
$$

Здесь операторы $\underset{\sigma \vec{k} s}{a}$ относятся к уничтожению элементарных возбуждений в валентных зонах с энергиями $\varepsilon_{\sigma}(\vec{k}), \mu-$ химический потенциал. Константы эффективного межзонного взаимодействия элемен- 
тарных возбуждений определены через коэффициенты линейного межзонного электрон-фононного взаимодействия $V_{\sigma \sigma^{\prime}}$ и фононные частоты $\omega_{\rightarrow}$ следующим образом

k

$$
W_{\sigma \sigma^{\prime}}\left(\vec{k}, \overrightarrow{k^{\prime}}\right)=\frac{\left|V_{\sigma \sigma^{\prime}}\left(\vec{k}-\overrightarrow{k^{\prime}}\right)\right|^{2 \hbar \omega_{\vec{k}-k^{\prime}}}}{\left[\varepsilon_{\sigma}(\vec{k})-\varepsilon_{\sigma^{\prime}}\left(\overrightarrow{k^{\prime}}\right)\right]^{2}-\left(\hbar_{\omega_{\vec{k}-k^{\prime}}}\right)^{2}}
$$

Взаимодействие, связанное с (2), описывает под поверхностью Ферми рассеяние пар дырок и над поверхностью Ферми пар электронов из одной зоны в другую. В отличие от куперовских пар активные волновые векторы не ограничены здесь узкой областью вокруг поверхности Ферми.

Далее для упрощенной интерполяции будет рассматриваться двухзонная модель с законами дисперсии типа $\left(m_{1,2}>0\right)$

$$
\begin{aligned}
& \varepsilon_{1}(\vec{k})=-\frac{\hbar^{2}}{2 m_{1}}\left(k_{x}^{2}+k_{y}^{2}\right) \\
& \varepsilon_{2}(\vec{k})=-E_{0}-\frac{\hbar^{2}}{2 m_{2}}\left(k_{x}^{2}+k_{y}^{2}\right),
\end{aligned}
$$

где $\vec{k}$ - отсчитывается от соответствующей $(X ; S)$ симметричной точки зоны Бриллюэна и полагается, что $m_{2}>m_{1}$, т. е. зона тяжелых дырок лежит глубже. Истинный электронный спектр высокотемпературных сверхпроводников моделируется при этом даже в актуальной области весьма грубо, но это не имеет здесь особого значения. Плотности состояний для двухмерного спектра (3) можно записать в виде $-\varrho_{1,2}=-\frac{v k_{z} m_{1,2}}{2 \pi^{2} \hbar^{2}} n, \quad$ где $v-$ объем элементарной ячейки, $k_{z} \simeq \pi / a_{z}$, а $n$ - число эквивалентных долин.

Гамильтониан (1) можно диагонализовать четырехпараметрическим преобразованием Боголюбова. Для двух щелевых параметров порядка

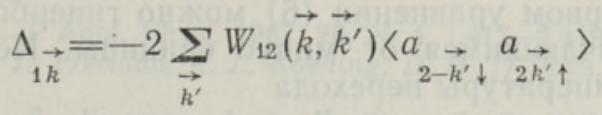

$$
\begin{aligned}
& \underset{2 \vec{k}}{\Delta_{\vec{k}}}=2 \underset{\overrightarrow{k^{\prime}}}{\sum_{21}} W_{2}\left(\vec{k}, \vec{k}^{\prime}\right)\left\langle a_{1-\vec{k}^{\prime} \downarrow} a_{1 \vec{k}^{\prime} \uparrow}\right\rangle,
\end{aligned}
$$

связанных с аномальными внутризонными средними, индуцируемыми взаимно межзонной связью получается система уравнений

$$
\begin{aligned}
& \Delta_{1 \vec{k}}=\sum_{\overrightarrow{k^{\prime}}} W_{12}\left(\vec{k}, \vec{k}^{\prime}\right) \Delta_{2 k^{\prime}} \frac{\left(1-2 f_{2}\left(\vec{k}^{\prime}\right)\right)}{\left\{\left[\varepsilon_{2}\left(\overrightarrow{k^{\prime}}\right)-\mu\right]^{2}+\Delta_{2 \vec{k}^{\prime}}^{2}\right\}^{1 / 2}} \\
& \underset{2 \rightarrow \vec{k}}{\Delta_{2 k}}=\sum_{\overrightarrow{k^{\prime}}} W_{21}\left(\vec{k}, \vec{k}^{\prime}\right) \Delta_{1 k^{\prime}} \frac{\left(1-2 f_{1}\left(\vec{k}^{\prime}\right)\right)}{\left\{\left[\varepsilon_{1}\left(\overrightarrow{k^{\prime}}\right)-\mu\right]^{2}+\Delta_{1 \vec{k}^{\prime}}^{2}\right\}^{1 / 2}} .
\end{aligned}
$$

Здесь $f_{1,2}$ - фермиевские функции распределения (ср. [ $\left.\left.{ }^{31}\right]\right)$. Рассмотрим в дальнейшем случай, когда $-E_{0}<\mu<0$, т. е. химический потенциал лежит над максимумом зоны тяжелых дырок в пределах первой 
зоны. Тем самым исключаются корреляции между электронами в качестве механизма сверхпроводимости $(W)$ в данной модели и дальнейшее основано на межзонном взаимодействии дырок.

Сделаем обычное (в данном случае, видимо, еще менее обоснованное, чем в БКШ-теории) предположение, что $W\left(\vec{k}, \vec{k}^{\prime}\right)$ является константной в области энергии от $\mu=-\zeta$ до энергии обрезания $E_{c}$. Понятие и фиксация последней требует специального обсуждения, что достаточно сложно. Физически $E_{c}$ должно определяться энергией, при которой скорость дырок становится столь большой, что за время жизни они успевают разлететься на расстояние большее корреляционной длины. На основании данных расчетов $\left[{ }^{4-10}\right]$ можно оценить порядок $E_{\mathrm{c}}-\zeta \simeq 1$ эВ

Анализ показывает, что системе (5) можно удовлетворить при положительном значении $W$. Это является характерным преимуществом межзонной модели.

Оценим температуру сверхпроводящего фазового перехода. В указанных приближениях из (5) получим систему

$$
\begin{gathered}
\beta=-W_{Q_{2}} \int_{\zeta-E_{0}}^{\zeta-E_{c}} \frac{d x}{x} \text { th } \frac{x}{2 k_{B} T_{c}} \\
\beta^{-1}=-W_{Q_{1}} \int_{0}^{\zeta-E_{c}} \frac{d x}{x} \text { th } \frac{x}{2 k_{B} T_{c}},
\end{gathered}
$$

где $\beta$ - предел отношения щелей при их стремлении к нулю, если $T \rightarrow T_{c}$.

Из анализа системы (6) следует, что с $\zeta \rightarrow E_{0}$ температура перехода $T_{c}$ существенно повышается. Отсюда вытекает, что для возникновения высокотемпературной сверхпроводимости требуется подходящий сдвиг уровня Ферми, достигаемый на опыте легированием $\mathrm{La}_{2} \mathrm{CuO}_{4}$ примесями типа $\mathrm{Ba}\left[{ }^{4,5,11}\right]$ и в $\mathrm{Y}_{1} \mathrm{Ba}_{2} \mathrm{Cu}_{3} \mathrm{O}_{7-\delta}$, видимо, созданием вакансий кислорода (ср. $\left.\left[{ }^{29}\right]\right)$. Далее положим $\zeta$ настолько сдвинутым относительно $E_{0}$, что в первом уравнении (6) можно гиперболический тангенс считать мало отличающимся от минус единицы. Исключая затем $\beta$, получим оценку температуры перехода

$$
k_{B} T_{c}=1,14\left(E_{c}-\zeta\right) e^{-1 / \lambda},
$$

где

$$
\lambda=W^{2} \varrho_{1} \varrho_{2} \ln \frac{E_{c}-\zeta}{E_{0}-\zeta} .
$$

Эта формула напоминает по структуре в некоторой мере результат БКШ. Но масштаб обрезаемых энергий здесь электронный. Межзонная константа взаимодействия имеет порядок $W \sim V^{2} \hbar \omega_{0}\left[\bar{E}^{2}-\left(\hbar \omega_{0}\right)^{2}\right]^{-1}$. Учитывая обычный порядок линейного электрон-фононного взаимодействия в ионных соединениях, для $V$ можно взять 0,45 эВ. Величина $\hbar \omega_{0}$ отвечает энергии деформационных колебаний кислородо-медной связи и, согласно [ $\left.{ }^{11}\right], \hbar \omega_{0} \sim 0,1$ эВ. Для произведения плотностей состояний реальна оценка $\varrho_{1} \varrho_{2} \approx 8(э \mathrm{~B})^{-2}$ ср. $\left[{ }^{8}\right]$ и $\bar{E} \approx 0,4$ эВ. Находим $W \approx 0,135$ эВ. Учтя еще, что $\ln \frac{E_{\mathrm{c}}-\zeta}{E_{0}-\zeta} \approx 2$, получаем в итоге $e^{-1 / \lambda} \sim 0,03$. Это означает, что поскольку $\left(E_{\mathrm{c}}-\xi\right) \sim 1$ эВ, то рассматриваемый механизм может обеспечить температуры сверхпроводящего перехода вплоть до комнатных, 
Следует заметить, что формула (7) содержит другого типа источник изотопического эффекта в сравнении с БКШ-теорией, несмотря на электрон-фононную природу исходного взаимодействия. В (7) массы атомов входят непосредственно через $W$, а в теории БКШ изотопический эффект проистекает от характеристической энергии фононного порядка, стоящей на месте $\left(E_{c}-\xi\right)$. Грубая оценка на основании $(2)$, (7) и (8) дает при изменении массы ячейки на $1 \%$ сдвиг $T_{c}$ порядка $5 \%$. Экспериментально в высокотемпературных сверхпроводниках изотопический эффект в $T_{c}$, хотя и наблюдается, но он значительно слабее, чем в системах, описываемых обычной БКШ-теорией $\left[{ }^{34,35}\right]$. Малость изотопического эффекта в высокотемпературных сверхпроводниках может быть связана и с нефононным вкладом в $W$ от собственноэлектронных межзонных корреляций носителей.

Число наблюдаемых щелей в спектре определяется в настоящей схеме числом электронных зон, пересекающих уровень Ферми. Теория допускает разброс значений $2 \Delta_{1,2}(T=0) / k_{B} T_{c} \quad$ в интервале $1-10$ при разумных ограничениях на $\varrho_{1,2}, W$.

Естественно, подход данного сообщения носит качественный характер и количественно груб. В частности, мы игнорировали кулоновское отталкивание дырок. Уточнению подлежит также вклад отдельных колебательных ветвей и межзонное электрон-фононное взаимодействие. чания.

Авторы благодарны В. Хижнякову и А. Пищеву за полезные заме-

\section{Л ИТ Р РА Т У РА}

1. Bednorz, J. G., Müller, K. A. // Z. Phys. B, 1986, 64, № 2, 189-193.

2. $W u, M$. K., et al. // Phys. Rev. Lett., 1987, 58, № 9, 908-910.

3. Ovshinski, S. R. et al. // Phys. Rev. Lett., 1987, 58, № 24, 2579-2581.

4. Mattheiss, L. F. // Phys. Rev. Lett., 1987, 58, № 10, 1028-1030.

5. Yu, J., Freeman, A. J., Xu, J.-H. //Phys. Rev. Lett., 1987, 58, № 10, 1035-1037.

6. Takegahara, K., Harima, H., Yanase, A. // Jap. J. Appl. Phys., 1987, 26, № 4, L352-L354.

7. Oguchi, T. // Jap. J. Appl. Phys., 1987, 26, № 4, L417-L419.

8. Massidda, S., Yu, J., Freeman, A. J., Koelling, D. D. // Phys. Lett. A, 1987, 122, № 3, $4,198-202$.

9. Yu, J., Massidda, S., Freeman, A. J., Koelling, D. D. // Phys. Lett. A, 1987, 122, № 3, 4, 203-208.

10. Ching, W. Y. et al. // Phys. Rev. Lett., 1987, 59, № 12, 1333-1336.

11. Weber, W. // Phys. Rev. Lett., 1987, 58, № 13, 1371-1374.

12. Kopaev, Yu. V., Rusinov, A. I. // Phys. Lett. A, 1987, 121, № 6, 300-304.

13. Plakida, N. M., Aksenov, V. L., Drechler, S. L. Preprint JINR, E17-87-198, Dubna, 1987.

14. Кулик Н. О. // ФНТ, 1987, 13, № 8, 879-883.

15. Matsuura, T., Miyake, K. // Jap. J. Appl. Phys., 1987, 26, № 4, L407-L409.

16. Fukuyama, H., Yosida, K. // Jap. J. Appl. Phys., 1987, 26, № 4, L371-L373.

17. Kivelson, S. A., Rokhsar, D. S., Sethna, J. P. I/ Phys. Rev. B, 1987, 35, № 16, $8865-8868$.

18. Parmenter, R. H. // Phys. Rev. Lett., 1987, 59, № 8, 923-925.

19. Ruckenstein, A. E., Hirchfeld, P. J., Appel, J. // Phys. Rev. B, 1987, 36, № 1, $857-860$.

20. Emery, V. J. // Phys. Rev. Lett., 1987, 58, № 26, 2794-2797.

21. Anderson, P. W., Baskaran, G., Zou, Z., Hsu, T. // Phys. Rev. Lett., 1987, 58, № 26, $2790-2793$.

22. Ruvalds, J. // Phys, Rev. B, 1987, 35, № 16, 8869-8872.

23. Hirsch, J. E. // Phys. Rev. B, 1987, 35, № 16, 8726-8729.

24. Chen, J. T., McEwan, C. J., Wenger, L. E., Logothetis, E. M. // Phys. Rev. B, 1987, 35 , № $13,7124-7125$.

25. Лущик Ч. Б. и др. // Пнсьма в ЖЭТФ, 1987. 46, вып. 3, 122-124.

26. Янсон И. К. и др. // ФНТ, 1987, 13, № 5, 557-560.

27. Kristoffel, N., Konsin, P. // Ferroelectrics, 1973, 6, 3-12; 1978, 21, № 1-4, 477-479. 
28. Берсукер И. Б., Полингер В. З. Вибронные взаимодействия в молекулах и кристаллах. М., Наука, 1983.

29. Габович А. М., Моисеев Д. П. // УФН, 1986, 150, вып. 4, 599-623.

30. Birgeneau, R. J. et al. // Phys. Rev. Lett., 1987, 59, № 12, 1329-1332.

31. Kondo, J. // Prog. Theor. Phys., 1963, 29, № 1, 1-9.

32. Батыев Э. Г., Борисюк В. А. // ЖЭТФ, 1986, 90, вып. 2, 558-568.

33. Мойжес Б. Я., Супрун С. Г. // ФТТ, 1987, 29, вып. 2, 441-448.

34. Faltens, T. A. et al. // Phys. Rev. Lett, 1987, 59, № 8, 915-918.

35. Batlogg, B. et al. // Phys. Rev. Lett., 1987, 59, № 8, 912-914.

\author{
Институт физики \\ Академии наук Эстонской ССР
}

Поступила в редакцию 9/XI 1987

\title{
P. KONSIN, N. KRISTOFFEL, T. ORD \\ KORGETEMPERATUURILISE ULIJUHTIVUSE TSOONIDEVAHELISE ELEKTRON-FOONON-MEHHANISMI VOIMALIKKUSEST
}

On välja pakutud kõrgetemperatuurilise ülijuhtivuse võimalik mehhanism, mis põhineb kattuvate aukjuhtivustsoonide elektron-foonon-interaktsioonil.

\section{P. KONSIN, N. KRISTOFFEL and T. ORD}

\section{ON THE POSSIBILITY OF THE INTERBAND ELECTRON-PHONON MECHANISM OF HIGH-TEMPERATURE SUPERCONDUCTIVITY}

A possible mechanism of high-temperature superconductivity based on the interband electron-phonon interaction of hole conductivity overlapping bands is proposed. 